\title{
1-S09-4 Symposium
}

\section{Mechanisms of cell proliferation through primary cilium}

\author{
Masaki Saito ${ }^{1}$, Wataru Otsu², Ching-Hwa Sung ${ }^{2}$
}

${ }^{1}$ Dept. Mol. Pharmacol., Tohoku Univ. Grad. Sch. Med., ${ }^{2}$ Dept. Ophthalmol., Weill Med. Col. Cornell Univ.

Primary cilium is a nonmotile sensory organelle that possesses selective membrane receptors. The cilium is dynamically regulated in a cell cycle-dependent manner; it is displayed at the $G_{0} / G_{1}$ phase of many cell types, including neural progenitor cells, and resorbed prior to the $S$ phase. The ciliary dynamics has pivotal roles in development of many tissues/organs. However, the molecular mechanism how the cilium controls cell proliferation remains largely unknown. We found that IGF-1 was one of the growth factors that promoted proliferation of neural progenitors. Tctex-1, a light chain of cytoplasmic dynein that plays a key role in ciliary resorption, can be free from dynein complex when it is phosphorylated at Thr 94 . We also found that phospho-Tctex-1 was enriched at the ciliary base in the cells. Molecular approaches analyzed in an immortalized retinal pigment epithelial cell line revealed the physical and functional interaction of phospho-Tctex-1 with the regulators of actin dynamics such as annexin A2, Arp2/3 complex and Cdc42, which promoted branched actin polymerization and dynamin- and clathrin-dependent endocytosis at peri-ciliary region. Our study demonstrated that these mechanisms collectively regulate ciliary resorption and proliferation of neural progenitors. 